Methodology Female patients aged $\geq 18$ years with histologically/cytologically confirmed metastatic/unresectable ovarian cancer, measurable disease per Response Evaluation Criteria in Solid Tumors (RECIST v1.1), Eastern Cooperative Oncology Group (ECOG) performance status $0 / 1$, and 3 prior lines of therapy were enrolled. Patients received lenvatinib $20 \mathrm{mg}$ daily plus pembrolizumab $200 \mathrm{mg}$ every 3 weeks for 35 cycles, or until confirmed disease progression or unacceptable toxicity. Primary endpoints were objective response rate (ORR; response assessed every 9 weeks for 54 weeks, then every 12 weeks, by blinded independent central review per RECIST v1.1) and safety. Secondary endpoints included disease control rate, duration of response, and progression-free survival.

Results 31 patients with ovarian cancer received $\geq 1$ dose of lenvatinib plus pembrolizumab in LEAP-005 (median age 62 years [range 40-76]); median study follow-up was 7.8 months (range, 4.6-12.4) as of April 10, 2020. ORR was 32\% (95\% confidence interval, 17-51); other efficacy endpoints were also favorable (table 1). Treatment-related adverse events occurred in 29 (94\%) patients (table 1).

Conclusion Lenvatinib plus pembrolizumab demonstrated encouraging efficacy and manageable safety in patients with heavily pretreated ovarian cancer, including those with prior platinum failure and those with previous bevacizumab exposure.

Disclosures Antonio González-Martín: Advisory/Consultancy: Amgen, AstraZeneca, Clovis Oncology, Genmab, GSK, ImmunoGen, Mersana, Merck Sharp \& Dohme, Novartis, Oncoinvent, Pfizer/Merck, PharmaMar, Roche, Sotio; Speaker Bureau: AstraZeneca, PharmaMar, Roche, GSK; Research Grant/Funding: Roche, TESARO: A GSK Company; Travel/Accommodation/Expenses: AstraZeneca, PharmaMar, Roche, TESARO: A GSK Company

Hyun Cheol Chung: Grants/Research Support: Lilly, GSK, MSD, Merck-Serono, BMS/Ono, Taiho, Amgen, Beigene, Incyte; Honoraria: Merck-Serono, Lilly/Foundation Medicine; Consultation: Taiho, Celltrion, MSD, Lilly, Quintiles, BMS, Merck-Serono, Gloria, Beigene, Amgen, Zymework

\begin{tabular}{|c|c|c|}
\hline & & $\begin{array}{c}\text { Lenvatinib }+ \\
\text { Pembrolizumab }(n=31)\end{array}$ \\
\hline \multicolumn{3}{|l|}{ Efficacy } \\
\hline \multicolumn{2}{|c|}{ Confirmed ORR, \% $(95 \% \mathrm{CI})$} & $32(17-51)$ \\
\hline \multicolumn{2}{|c|}{ Disease control rate, ${ }^{a} \%(95 \% \mathrm{CI})$} & $74(55-88)$ \\
\hline \multicolumn{2}{|c|}{ Duration of response, median (range) months } & $\mathrm{NR}(1.5+$ to $7.9+)$ \\
\hline \multicolumn{2}{|c|}{ Progression-free survival, median $(95 \% \mathrm{CI})$ months } & $4.4(4.0-8.5)$ \\
\hline \multicolumn{3}{|c|}{ Responders by prior therapy, $\mathrm{n}(\%)^{\mathrm{b}}$} \\
\hline \multicolumn{2}{|c|}{ Platinum refractory/resistant $(\mathrm{n}=\mathbf{2 5})$} & $6(24)$ \\
\hline \multicolumn{2}{|c|}{ Bevacizumab exposed ( $\mathbf{n}=19)$} & $4(21)$ \\
\hline \multicolumn{2}{|c|}{ Treatment-related AEs, n (\%) } & $29(94)$ \\
\hline \multicolumn{2}{|c|}{ Grade 3-5 treatment-related AEs } & $21(68)$ \\
\hline \multicolumn{2}{|c|}{ Treatment-related AEs leading to death } & $1(3)^{\mathrm{c}}$ \\
\hline \multicolumn{2}{|c|}{ Treatment-related AEs resulting in treatment discontinuation } & $4(13)$ \\
\hline \multicolumn{3}{|c|}{ Treatment-related AEs occurring in $\geq 10$ patients } \\
\hline & Any Grade ${ }^{d}$ & Grade 3 \\
\hline Hypertension & $17(55)$ & $6(19)$ \\
\hline Fatigue & $13(42)$ & $3(10)$ \\
\hline Hypothyroidism & $13(42)$ & 0 \\
\hline Decreased appetite & $12(39)$ & 0 \\
\hline Diarrhoea & $12(39)$ & $1(3)$ \\
\hline Proteinuria & $10(32)$ & $2(6)$ \\
\hline \multicolumn{3}{|c|}{ 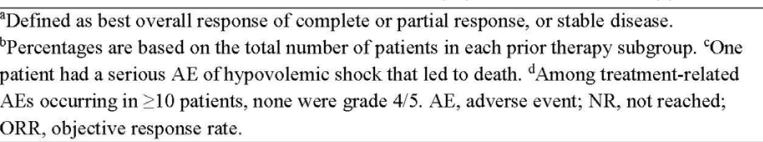 } \\
\hline
\end{tabular}

Esma Saada-Bouzid: Advisory/Consultancy: BMS, MSD; Travel/Accommodation: AstraZeneca, BMS, MSD

Eduardo Yanez: Consultant/Advisory Board: BMS, Merck Serono; Research Grant: Amgen, Pfizer, Astellas, BMS, Roche, Abbvie, MSD

Hélène Senellart: Nothing to disclose.

Philippe A. Cassier: Honoraria: Novartis, Roche/Genentech, Blueprint Medicines, Amgen, AstraZeneca; Research Funding: Novartis, Roche/Genentech, Lilly, Blueprint Medicines, Bayer, AstraZeneca, Celgene, Plexxikon, Abbvie, BMS, Merck Serono, MSD, Taiho Pharmaceutical, Toray Industries, Transgene, Loxo, GSK, Innate Pharma, Janssen (all paid to institution); Travel, Accomodations, Expenses: Roche, Amgen, Novartis, BMS, MSD, Netris Pharma.

Bristi Basu: Consultancy: GenMab (paid to institution); Advisory Board: Roche, Eisai Europe Limited, research grant from Celgene Ltd (all paid to institution); Speakers Bureau: Eisai Europe Ltd (paid to institution); Travel and Registration for Congress: Bayer.

Razi Ghori: employee of Merck Sharp \& Dohme Corp., a subsidiary of Merck \& Co., Inc., Kenilworth, NJ, USA

Peter Kubiak: employee of Eisai Inc., Woodcliff Lake, NJ, USA

Alan Smith: employee of Eisai Ltd., Hatfield, UK

Kevin Norwood: employee of Merck Sharp \& Dohme Corp., a subsidiary of Merck \& Co., Inc., Kenilworth, NJ, USA

Zarnie Lwin: Received honoraria for consulting, advisory role or travel sponsorship from AbbVie; AstraZeneca; BMS; Roche; and Merck

\section{THE EVOLVING ROLE OF PARP INHIBITORS IN NEWLY DIAGNOSED ADVANCED OVARIAN CANCER: THE EFFECT OF ONLINE EDUCATION ON CLINICIAN KNOWLEDGE, COMPETENCE AND CONFIDENCE}

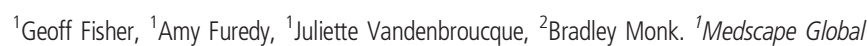
Education, New York, United States; ${ }^{2}$ University of Arizona College of Medicine, Phoenix, United States

\subsection{6/ijgc-2020-ESG0.117}

Introduction/Background The treatment of newly diagnosed advanced ovarian cancer is changing rapidly with the expanded use of PARP inhibitors. This study determined whether online continuing medical education could improve the knowledge, competence and confidence of oncologists and obstetricians/gynaecologists (obs/gyns) with regard to the application of PARP inhibitors in this setting.

Methodology A 30-minute online video panel discussion with synchronized slides was launched for physicians outside the USA in November 2019. Data was collected to January 2020. Educational effect was assessed with repeated-pairs pre-/post-activity, where individual participants served as their own control. 3 multiple-choice, knowledge questions and 1 self-efficacy, 5-point Likert scale confidence question were analyzed. Chi-squared test assessed pre- to post-activity change $(5 \%$ significance level, $\mathrm{P}<.05)$. Magnitude of change in total number of correct responses overall, and for each question, were determined with Cramer's V $(<.06=$ Modest,$\quad 0.06-0.15=$ Noticeable, $.16-.26=$ Consider able, $>.26=$ Extensive).

Results 157 oncologists and 152 obs/gyns completed pre- and post-activity questions. A positive educational effect was 
observed for both oncologists (considerable effect, $V=.159$, $\mathrm{P}<.0001$; with average $\%$ of correct responses increasing from 59 to $74 \%$ ) and obs/gyns (noticeable effect, $\mathrm{V}=.101, \mathrm{P}<.01$; average $\%$ of correct responses increasing from 48 to $58 \%$ ). Participants with $3 / 3$ correct answers increased from pre- to post-activity (16 to $44 \%$ for oncologists and 11 to $32 \%$ for obs/gyns). Improvements in $\%$ of correct responses post-activity were seen for questions on identifying data from PRIMA trial of niraparib (oncologists: 18 to 48\%; obs/gyns 16 to 41\%) and the PAOLA trial data for olaparib (oncologists: 68 to $80 \%$; obs/gyns 53 to 59\%). Participants had a good baseline understanding of the correct treatment approach for a patient presenting with HRD +ve advanced ovarian cancer $(90 \%$ oncologists, 74\% obs/gyns with the correct answer), although no increases were observed post-activity. Confidence in the ability to integrate PARP inhibitors into practice improved post-activity (total average confidence shift: 14\% for oncologists and 29\% for obs/gyns). $47 \%$ of all participants stated they would modify treatment plans as a result of participation in the activity.

Conclusion This on-demand, online video panel discussion resulted in a positive educational impact. However, education gaps remain evident, especially amongst obs/gyns. Online medical education, increasingly important during the COVID-19 pandemic, is valuable in supporting implementation of new treatment strategies and identifying areas of continued educational need.

Disclosures Supported by an independent education grant from GlaxoSmithKline

Geoff Fisher, Amy Furedy, Juliette Vandenbroucque have no relevant financial disclosures

Bradley Monk has served as an advisor or consultant for a range of pharmaceutical companies including AstraZeneca, Clovis and GlaxoSmithKline.

\section{CLINICAL CHARACTERISTICS AND PROGNOSIS OF OVARIAN CLEAR CELL CARCINOMA: A 10-YEAR RETROSPECTIVE STUDY}

${ }^{1}$ Chenchen Zhu, ${ }^{2}$ Jing Zhu, ${ }^{1}$ Shuangfeng Chen, ${ }^{1}$ Qingqing Zhou, ${ }^{2}$ Hanyuan Liu, ${ }^{2}$ Sisi Deng, ${ }^{2}$ Luwen Liu, ${ }^{2}$ Ying Zhou. 'Department of Obstetrics and Gynecology, Anhui Provincial Hospital, Anhui Medical University; ${ }^{2}$ Department of Obstetrics and Gynecology, The First Affiliated Hospital of Ustc, Division of Life Sciences and Medicine, University of Science and Technology of China

\subsection{6/ijgc-2020-ESG0.118}

Introduction/Background Ovarian clear cell carcinoma (OCCC) is a special subtype of epithelial ovarian carcinoma with unique characteristics and no specific tumour markers. Due to the inherent chemoresistance, there are no effective chemotherapy regimen for OCCC, resulting in the extremely poor prognosis of patients, especially at advanced stage. Therefore, the purpose of our research is to investigate the clinical characteristics and outcomes of ovarian clear cell carcinoma (OCCC) and to provide additional supporting evidence to aid in the clinical diagnosis and management.

Methodology This was a retrospective study investigating the clinical characteristics and survival outcomes of 87 patients with OCCC treated at The First Affiliated Hospital of University of Science and Technology of China (USTC), between January 2010 and March 2020. Survival analysis was also performed on 179 patients with OCCC diagnosed between 1975 and 2017, obtained from the Surveillance, Epidemiology and End Results (SEER) cancer registry database.

Results The median age of study participants was $49.28 \pm$ 9.8 years old, with $74.71 \%$ diagnosed at an early stage. Median CA125 level was $607.26 \mathrm{IU} / \mathrm{mL}$, with $23.94 \%$ having a normal CA125 level. 16 patients (18.39\%) had coexisting endometriosis and 8 patients (9.2\%) had a preoperative history or developed postoperative complications of venous thromboembolism (VTE). Surgical staging procedures were performed on 65 patients and cytoreduction was performed on 22 patients, among whom 17 patients received optimal cytoreduction. 67 patients (77.01\%) underwent lymphadenectomy, and only $3(4.48 \%)$ were found to have positive lymph nodes. Positive HNF1 $\beta$, and negative WT-1, ER, and PR are reliable immunohistochemical indicators of OCCC. Patients diagnosed at an early stage had higher 3 year overall survival (OS) $(89.47 \%$ vs. $44.44 \%)$ and progression-free survival (PFS) rates (78.95\% vs. $22.22 \%)$ than those with advanced stage OCCC at diagnosis. CA199 (P = $0.025)$ and ascites $(\mathrm{P}=0.001)$ were significantly associated with OS, while HE4 $(\mathrm{P}=0.027)$ and ascites $(\mathrm{P}=0.001)$ were significantly associated with PFS. Analysis of data from the SEER database showed that the presence of positive lymph nodes is also an independent prognostic factor for OS $(\mathrm{P}=0.001)$.

Conclusion OCCC often presents at an early stage and young age with a mild elevation in CA125 level. CA199, HE4, massive ascites and positive lymph node are independent prognostic factors for overall survival in OCCC.

Disclosures This work was supported by the National Natural Science Foundation of China $(81872110,81902632)$, National Key Research and Development Program (2018YFC1003900), Anhui Provincial Key Research and Development Program (1704a0802151). The funders had no role in the study design, data collection and analysis, decision to publish, or preparation of the manuscript. No conflicts of interest to disclose.

\section{CHARLSON COMORBIDITY INDEX AS A FACTOR IMPACTING SURVIVAL AMONG OVARIAN CANCER PATIENTS - RESULTS FROM A SYSTEMATIC REVIEW}

${ }^{1}$ Smita Asthana, ${ }^{2}$ Rahul Modi. 'National Institute of Cancer Prevention and Research (Nicpr); Division of Epidemiology and Biostatistics; ${ }^{2}$ All India Institute of Medical Sciences (Aiims), Rishikesh; Gynaecological Oncology

\subsection{6/ijgc-2020-ESGO.119}

Introduction/Background There are a few scattered primary level studies from various regions of the globe proving impact of co-morbidities on survival in ovarian cancer patients. In last 20 years, there has been improvement in survival among ovarian cancer patients. Radical surgical approaches and advancements in chemotherapeutic agents have primarily contributed for same. Pre-existing uncontrolled co-morbidities impact ovarian cancer survival directly and indirectly. This affects the performance status of the patient leading to delay in treatment or aversion from radical surgical approaches thereby not achieving the goal of optimal treatment. It may also lead to a less aggressive chemotherapeutic modifications of using lower doses or single agent chemotherapy. The objective of this study was to systematically review the literature and summarize prevalence of various comorbidities with evaluation of impact of the Charlson Co-morbidity Index (CCI) on survival in ovarian cancer patients. 